\title{
Automatic Synthesis of Control Programs by Combination of Learning and Problem Solving Methods (Extended Abstract)
}

\author{
Wolfgang Müller and Fritz Wysotzki \\ Fraunhofer-Institute for Information and Data Processing \\ Branch Lab for Process Optimization \\ Kurstr. 33, D-10117 Berlin, email: wmueller@epo.iitb.fhg.de
}

\section{Introduction}

An especially important field of today's research in Machine Learning is the control of complicated dynamical processes in environment and technology which cannot (or only partly) be modelled mathematically. Seen from the cognitive point of view there are close similarities to the problem of skill acquisition.

A series of learning methods for generating control trajectories - in the state space of a process - for real time applications have been investigated in AI and Neural Information Technology in recent years (e.g. BOXES [3, 5], CART [2], ASE/ACE [1]). In general, these approaches have disadvantages in real time applications and their complexity may increase dramatically if more than two different control actions are to be applied. Furthermore an apriori splitting of the state space is needed for some of them.

This article outlines an approach for generating a series of optimal control actions by combining learning with simple problem solving methods, which has a suitable real time behaviour and can handle a larger number of different discrete control actions.

\section{Control of dynamic processes based on classification learning (learning control)}

This approach tackles the splitting problem of the state space into boxes as a task of classification learning. As class an optimal control action will be attached to points in the state space assuming a set of discrete ones to be available. Generally, the approach proposed in this paper consists of the following steps: (a) generation of a training data set: the optimal control action is not determined by an expert [8] but by a problem solver using an empirical evaluation function for process states having its extremum at the target (goal) area; (b) splitting the state space automatically into subareas with unique control actions attached by learning: this way one obtains a classifier which is able to assign an optimal control action to any point of the state space in extremely short execution time (the steps (a) and (b) can be carried out off-line, where time is not critical); (c) automatic generation of control trajectories in real time by applying the classifier recursively (see also $[8,4]$ ).

As an example control problem the roll axis stabilization (as reaction to disturbances) of a communication satellite in orbit with respect to its position to the earth axis was taken. Here the real control process was substituted by an 
appropriate simulator developed by MBB Munich within the ESPRIT-project Statlog [9] (see also [4]). The position manoeuvres are carried out by thruster torques delivering a set of discrete control actions the application of which consumes fuel. Therefore the control task is to hold the satellite axis in a certain small target interval of its attitude angle consuming as little fuel as possible (i.e. performing a small number of small control actions). The state space of the process is defined by the attitude angle $\phi$ of the satellite and its rate $\dot{\phi}$. The target area of control is some region around $\phi=0$ and $\dot{\phi}=0$ (some maximal value of $|\phi|$ is given which must not be exceeded). There are additional variables $q_{1}, q_{2}, \dot{q}_{1}, \dot{q}_{2}$ describing oscillations of the two solar generators of the satellite which influence the process states especially the rate $\phi$. They are treated as "hidden parameters" and their influence could be considered as noise or taken into account by including a history of preceding states in the definition of current state.

As a training set a set of $n$ points $m_{j} \in M, j=1,2, \ldots, n, m_{j}=\left(\phi_{j}, \dot{\phi}_{j}\right)$ in the two dimensional state space, was selected at random and to each of them an "optimal" control action $f_{j o p t}$ assigned. This action was chosen automatically from a given (finite) set of executable control actions $P_{f}=\left\{f_{1}, f_{2}, \ldots, f_{q}\right\}$ by an empiric evaluation function $F: X \rightarrow R$, which maps the state space $X$ into the set of real numbers. The applied control actions $f_{i}$ produce for each point $m_{j}$ a set $\bar{M}_{j}$ of temporary successor points

$$
\bar{m}_{j, i}=g\left(m_{j}, f_{i}\right), \bar{m}_{j, i} \in \bar{M}_{j}, i=(1,2, \ldots, q) .
$$

$\bar{M}_{j}$ is computed by the simulator, and must be found by experimentation with the process if no model is available. The optimal control action $f_{\text {opt }}$ for point $m_{j}$ is that $f_{i}$ for which the evaluation function at the next state generated by applying this action becomes a minimum:

$$
F\left(\bar{m}_{j o p t}\right)=\min _{i \in\{1, \ldots, q\}} F\left(\bar{m}_{j, i}\right)=\min _{i \in\{1, \ldots, q\}} F\left(g\left(m_{j}, f_{i}\right)\right)=F\left(g\left(m_{j}, f_{j o p t}\right)\right)
$$

By carrying out this procedure for all $m_{j} \in M$ a learning data set $M^{\prime}=$ $\left\{m_{j}, f_{j o p t}\right\}, j=1,2, \ldots, n$ is formed. This is gradient descent, known to give a local optimal solution in general but a global optimal solution, too, in the case of a convex evaluation function having exactly one extremal point (greedy behaviour).

For the current process a convex $F$ can be chosen. The simplest is

$$
F(\phi, \dot{\phi}, f)=\phi^{2}+\kappa \dot{\phi}^{2}+\varepsilon|f|,
$$

the sum of a weighted (squared) Euclidean distance from the origin and a weighted penalty function. $|f|$ stands for the value of the control torque and is proportional to the amount of fuel consumption. The weights $\kappa$ and $\varepsilon$ were optimized due to some criteria for achieving a good control performance (i.e. small amount of fuel consumption and some desirable properties of control curves). A satisfying parameter combination found after systematic experimentation is $\kappa=10^{-2}, \varepsilon=8 \cdot 10^{-10}$. These parameter values were also confirmed by theoretical considerations using a simple process model based on the equations of motion for $\phi$ and $\dot{\phi}$. 


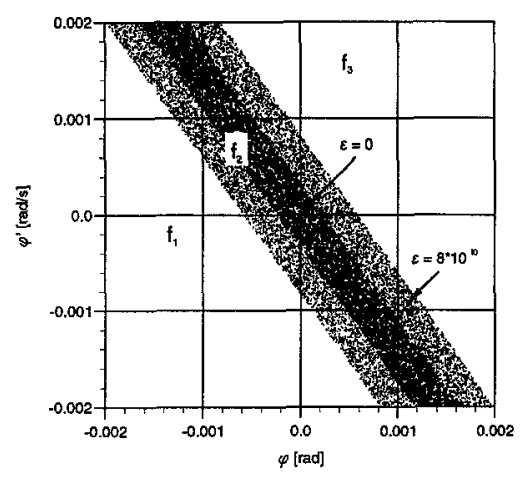

Fig.1. State space split into three subareas of different control actions, $f_{1}=20 \mathrm{Nm}$, $f_{2}=0 N m, f_{3}=-20 N m$

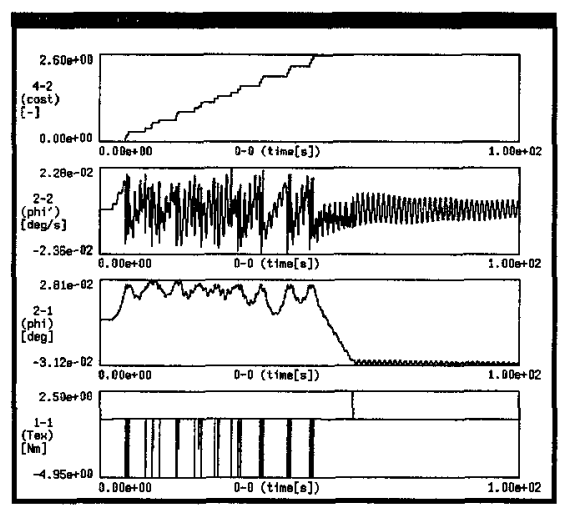

Fig.2. Plot over time of satellite's behaviour. 5 control torques $( \pm 5, \pm 2.5$, $0 \mathrm{Nm}$ ) used. Tex is the sum of disturbance and control torque

Figure 1 is the graphical representation of the state space after assigning an optimal control action to each point assuming three different control actions available ${ }^{1}$. The state space contains three homogeneous subareas due to different optimal control actions. Two different evaluation functions $\left(\varepsilon=0, \varepsilon=8.10^{-10}\right)$ are presented to demonstrate the effect of the penalty function. To the constructed training set the classification algorithms CAL 5 and DIPOL $[3,6,7]$ were applied to develop optimal decision functions. There were no significant differences in control performance. Of course other methods of classification learning can be applied, too. A learned classification function $\psi$ now delivers an optimal control trajectory from any state $s$ into the set of target states $S_{\text {goal }}$ by means of the recursion equation $\Psi(s)=$ if $s \in S_{\text {goal }}$ then $s$ else $\Psi\left(\psi_{s}(s)\right)$, where $\psi_{s}$ is the output (optimal control action) of the classifier $\psi$ for input state $s$ and $\psi_{s}(s)$ the result of appplication of $\psi_{s}$ to $s$ (the next state).

The classifiers developed by CAL 5 and DIPOL were built into the simulator instead of the original PD-controller and the simulation results were compared with those of the original controller.

\section{Results}

An example of simulated satellite's behaviour is shown in figure 2. The applied disturbance was $0.05 \mathrm{Nm}$ per $50 \mathrm{sec}$. The system is stabilisized by a decision tree controller developed by CAL 5 immediately after the disturbance has disappeared. The main results of a number of experiments (varying the type of $F$ and the generalization level of the classifiers) are: 1) The above introduced control method using learned classifications of process states by optimal control

\footnotetext{
${ }^{1}$ Note that applying a "control action" $f=0 \mathrm{Nm}$ (i.e. doing nothing) corresponds to leaving the system move according to its inherent dynamics governed by the equations of motion.
} 
actions shows better results in most cases than an appropriate "classical" PD -controller (better stabilization, smaller number of control actions, i.e. less fuel consumption). Experiments in which a state history of length 4 was included in the definition of a state and performed to take into account the influences of "hidden parameters" (oscillations of solar arrays) resulted in even better control performance of the classifier. Errors of the classifiers lead to some amount of suboptimality as it is expected. 2) The parameters of the evaluation function strongly influence the fuel consumption and the control performance. At present, experiments for optimization (learning) of these parameters are performed in our lab. 3) The more control actions in the range from $+20 \mathrm{Nm}$ to $-20 \mathrm{Nm}$ were used the better the control performance became. 4) In the case of more complicated processes having "forbidden regions" in the state space the definition of a convex evaluation function is no longer possible and more general problem solving methods have to be applied. This will be a topic of future research.

Acknowledgements: Thanks are due to $\mathrm{C}$. Bruehe for carrying out the experiments.

\section{References}

1. Barto, A. G, Sutton, R. S.,Anderson, C. W.: Neuronlike Adaptive Elements That Can Solve Diffcult Learning Control Problems, IEEE Transactions on Systems, Man and Cybernetics, Vol. SMC-13, Number 5, pp 835 - 846, 1983

2. Connell, M. G./Utgoff, P. E.: Learning to Control a Dynamic Physical System, AAAI 87, Seattle, pp $456-460,1987$

3. Michie, D.; Spiegelhalter, D.J.; Taylor, C.C.: Machine Learning, Neural and Statistical Classification. Ellis Horwood, Hertfordshire, U.K., 1994

4. Michie D. : Experiments in Rule Based Control, Beyond the Neural Pradigm for Machine Learning. Proc. of JSAI meeting, Tokyo, June 1991

5. Michie, D.,Chambers, R. A.: BOXES as a Model of Pattern-formation, Towards a Theoretical Biology, Vol. 1, Prolegomena, C. H. Waddington (Ed.), Edinburgh: Edinburgh Univ. Press, pp 206 - 215, 1968

6. Mueller,W.; Wysotzki,F.: Automatic Construction of Decision Trees for Classification in Annals of Operation Research 92, Moser, K., Schader M. (Eds) J.C. Baltzer AG Science Publishers, Wijdenes, Netherlands, 1994, pp.231-247

7. Schulmeister, B.; Wysotzki, F.: Piecewise Linear Classifier DIPOL92, Proc. of the ECML 94, 7th European Conference on ML, 1994

8. Sammut, C, et al : Learning to fly. Machine Learning, Proc. of the Ninth International Workshop ML 92, Morgan Kaufmann Publishers,San Mateo, CA

9. Statlog Deliverable 2.2 : Description and Specification of the Simulation Program of Spacecraft-Dynamics, ESPRIT Project 2170, Statlog (Comparative Testing of Statistical and Logical Learning), Munich, 1991 